\title{
Real-time FFT Analysis for Interferometric Sensors Multiplexing
}

\author{
Daniel Leandro, Mikel Bravo, Amaia Ortigosa and Manuel Lopez-Amo, Senior Member, IEEE.
}

\begin{abstract}
In this paper, a theoretical and experimental study of two interferometric sensor multiplexing schemes has been carried out by means of the fast Fourier transform (FFT) analysis. This work addresses one of the main drawbacks of photonic crystal fiber (PCF) sensors, that is, its multiplexing capability. Using a commercial optical interrogator combined with a simple FFT measurement technique, the simultaneous real-time monitoring of several PCF sensors is achieved. A theoretical analysis has been performed where simulations matched with the experimental results. For the experimental verification, highly birefringent (HiBi) fiber sections that operated as sensing elements were multiplexed and tested in two configurations. Due to the FFT analysis, both multiplexing schemes can be properly interrogated by monitoring the FFT phase change at the characteristic spatial-frequency of each sensor. For this purpose a commercial interrogator and a custom Matlab program were used for computing the FFT and for monitoring the FFT phase change in real-time $(1 \mathrm{~Hz})$.
\end{abstract}

Index Terms - Fiber loop mirror (FLM), Fourier transforms, multiplexing, optical fiber sensors, photonic crystal fiber (PCF).

\section{INTRODUCTION}

L IGHT interference in fiber optic structures is an interesting phenomena extensively used for different application such as optical filtering in optical communications applications [1], multi-wavelength fiber optic lasers [2] or in fiber optic sensor technologies [3]. In fiber optic sensing, some structures such as single-mode multi-mode single-mode (SMS), Mach-Zehnder, Michelson, Sagnac, Fox-Smith interferometric structures are used to develop point sensors. Commonly the interference is caused by the phase shift between two or more waves that finally interfere, for instance, in a fiber coupler, polarizer, fiber splice, etc.

Due to their special propagation characteristics, Photonic crystal fibers (PCF) are often used as sensing elements within interferometric structures. PCF based sensors are extensively used for chemical sensing or physical applications as

Manuscript received July, 29, 2014. This work was supported by the Spanish Government projects TEC2010-20224-C02-01, TEC2013-47264-C22-R, INNOCAMPUS, by the European SUDOE-Interreg Project ECOALMGT and the FEDER funds.

D. Leandro, M. Bravo, A. Ortigosa and M. Lopez-Amo, are with Department of Electric and Electronic Engineering, Public University of Navarra, Campus Arrosadia S/N, 31006, Navarra, Spain. (e-mail: daniel. leandro@unavarra.es).

Copyright (c) 2013 IEEE. Personal use of this material is permitted. However, permission to use this material for any other purposes must be obtained from the IEEE by sending a request to pubs-permissions@ieee.org. thoroughly explained in [4].

Due to the different properties of each interferometer, there are several effects that can be used as sensing parameter, mainly the phase shift. Typically, the phase information is measured by tracking the wavelength of an interference dip or alternatively by monitoring the intensity variation of a narrowband source at a fixed wavelength when the interference shifts [5].

Although the sensing ability of the PCF-based interferometers has been widely validated, nowadays few studies have focused on the multiplexing capability of these sensors [6], [7], [8] and [9]. The main drawbacks for the multiplexing are the high insertion losses and the low signal to noise ratios.

One of the proposed solutions use the fast Fourier transform (FFT) analysis. This is not a widely used technique although it provides a different point of view of the interference and in some cases it also offers useful and clear information to be used in sensing applications. As presented in [6], D. Barrera et al. use the FFT analysis for fiber optic interferometric sensors multiplexing. In that case, the interference of two SMS interferometers placed in series was analyzed by studying the FFT phase shift of each FFT frequency contribution due to the multi-mode interference.

In this work, two fiber optic multiplexing structures based on interferometers are proposed and analyzed theoretical and experimentally by means of the FFT analysis. Their sensing capability is also validated performing high precision strain measurements using sensors based on highly birefringent (HiBi) PCF.

\section{THEORETICAL CONSIDERATIONS AND PRINCIPLE OF OPERATION}

Fiber Sagnac interferometers have been extensively investigated both theoretically and experimentally due to their wide application in many fields such as fiber optic gyros and other fiber optic interferometric sensors [10]. The basic idea, firstly demonstrated by Georges Sagnac in 1913 [11], consists of injecting light from an optical source through a beam splitter such that it travels in two directions around a loop. If that loop is rotated, a phase shift between the propagating and counter propagating fields occurs, generating interference in the splitter. As a consequence, a simple Sagnac structure can detect rotation. A special situation comes up when the Sagnac structure, originally created in bulk optics, is adapted for fiber optic technology by using a common directional optical 


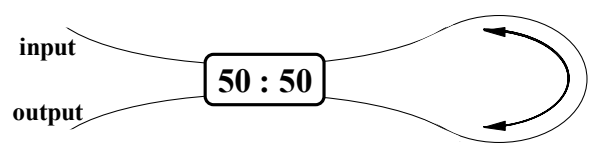

Fig. 1. Schematic depiction of the basic structure of a fiber loop mirror.

coupler (Figure 1) as beam splitter and a highly birefringent fiber into the loop. In this case, the cause of the interference is the phase shift between the polarization states. This special Sagnac structure is commonly known as high-birefringence fiber loop mirror (HiBi-FLM).

A fiber loop mirror consists of an optical fiber loop between the output ports of a directional coupler (Figure 1). D. B. Mortimore thoroughly studied this structure in [12] for HiBi fibers. As a result, considering birefringence in the mathematical development of the Jones matrix, the transmission coefficient between the input and output port is as follows.

$$
T=\left[\sin \theta \cos \left(2 \pi l \lambda_{0} / L_{B} \lambda\right)\right]^{2}
$$

Where $L_{B}$ refers to the fiber beat length, $l$ is the HiBi fiber length and $\theta$ denotes the difference between the rotation angle of the polarization states before and after the HiBi fiber section [13]. Thus, the dependence on the polarization angle limits the transmission coefficient. It should be noted that there is a reflection coefficient at the input port involved that is the complementary of the transmission coefficient $(R=1-T)$. Extrapolating these concepts to the fiber optic sensing field, the HiBi-FLM have great potential for fiber optic sensing as reported in [13].

In this paper, two structures for interferometric sensors multiplexing based on combinations of HiBi-FLM are theoretically and experimentally demonstrated.

\section{A. Star topology for multiplexing HiBi-FLMs using a 2xn optical coupler}

The first proposed scheme studies the interference behavior of multiple HiBi loops using a $2 \times n$ optical coupler $(n>2)$ as depicted in Figure 2. An interference combination of the multiple contributions due to the $n / 2$ loop interferometers is achieved by means of connecting the coupler output ports in pairs using HiBi fibers of different lengths. In this case, the transmission coefficient of the scheme will include $n / 2$ main contributions $\left(T_{\text {main }}\right)$ given by the fiber loops formed between the connected ports. In addition a large number of secondary contributions $\left(T_{s e c}\right)$ will be generated by the interaction of fields that do not form a loop.

$$
T=T_{\text {main }}+T_{\text {sec }}
$$

Using a single $2 \times n$ coupler and adjusting individually the polarization controller $\mathrm{PC}_{\mathrm{n}}$ of each loop, the main contributions given by the fiber loop mirrors (FLMs) can be maximized. Any other contribution due to fields travelling through different FLMs and interfering at the optical coupler will have an amplitude dependent on the relative angles of the different FLMs. The contribution will be located at the spatial

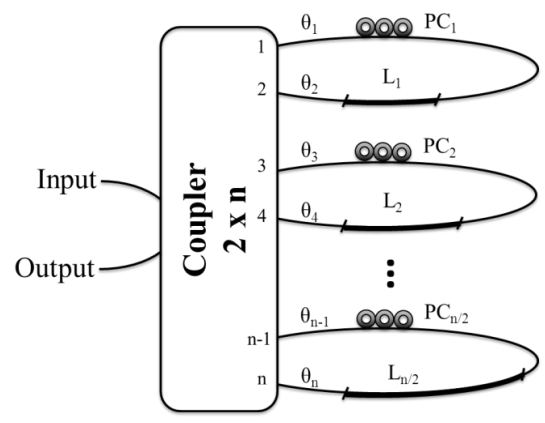

Fig. 2. Scheme of the HiBi-FLM interferometer using a $2 x n$ coupler.

frequencies given by the different lineal combinations of the spatial frequencies of the FLMs involved. Finally the contributions generated by fields travelling through different FLMs will depend on the phase shift given by the different HiBi-PCF sections present at the FLMs. Consequently, if its FFT phase is monitored, it will reflect the variations of several interferometric sensors at the same time.

Therefore those secondary contributions are not valid for sensing purposes and are not taken into account in this study, being monitored only the main contributions generated by the FLMs. In accordance to this, the relevant contributions to the transmission coefficient in a $2 x n$ coupler are as follows:

$$
\begin{aligned}
& T_{\text {main }}=\sum_{m=1}^{n / 2} T_{(2 m-1), 2 m}=T_{1,2}+T_{3,4}+\cdots+T_{n-1, n} \\
& T_{(2 m-1), 2 m}=\left[\sin \left(\theta_{(2 m-1), 2 m} \cos \left(2 \pi L_{m} \lambda_{0} / L_{B} \lambda+\varphi_{m}\right)\right]^{2}\right. \\
& m \in[1, n / 2]
\end{aligned}
$$

Where $T_{(2 m-1), 2 m}$ is the resulting transmission coefficient of the fiber loop mirror $m$ formed between the ports $2 m-1$ and $2 m, \theta_{(2 m-1), 2 m}$ is the relative angle $\left|\theta_{2 m-1}-\theta_{2 m}\right|$ given by the $\mathrm{PC}_{\mathrm{m}}$. It must be taken into account that the main contributions are proportional to $\sin \left(\theta_{(2 m-1), 2 m}\right)$ as stated in (1) so $\theta_{(2 m-1), 2 m}$ is experimentally set in order to obtain $\sin \left(\theta_{(2 m-1), 2 m}\right) \approx 1$. In the same manner, the amplitude of the secondary contributions is also dependent on the angles given by the PCs present at the involved loops. Experimentally, it implies that the secondary contributions which are dependent on several angles $\theta_{(2 m-1), 2 m}$ cannot be maximized. Accordingly, they are not comparable in terms of amplitude with the main ones, as can be experimentally seen in Fig. 4. $L_{m}$ is the length of the HiBi PCF fiber and $\varphi_{m}$ represents the birefringence-induced phase shift in the $m$-PCF fiber section. It is worth noticing that $\varphi_{m}$ is dependent only on the birefringence change of the $m$-fiber section so there is no cross-sensitivity between sensors as it will be experimentally verified in the next section. In order to monitor each interferometric sensor, the phase shift $\varphi_{m}$ is measured by using the FFT analysis.

In our experimental case a $2 \times 8$ optical coupler has been employed. Thus, the resulting transmission coefficient is the combination of the four different interference contributions. Figure 3 shows the simulated interference obtained when a $2 \times 8$ coupler is used. Four HiBi photonic crystal fibers with a beat length of approximately $3.45 \mathrm{~mm}$ and lengths of $0.3,0.8$, 


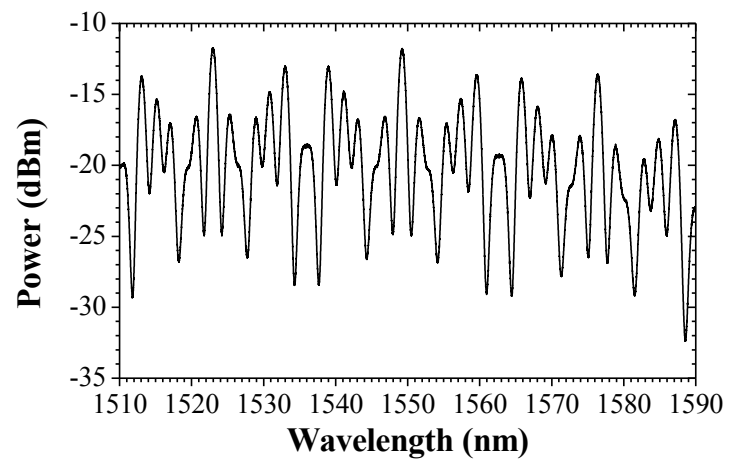

Fig. 3. Simulated optical spectrum for the 2x8 parallel multiplexed HiBi FLM.
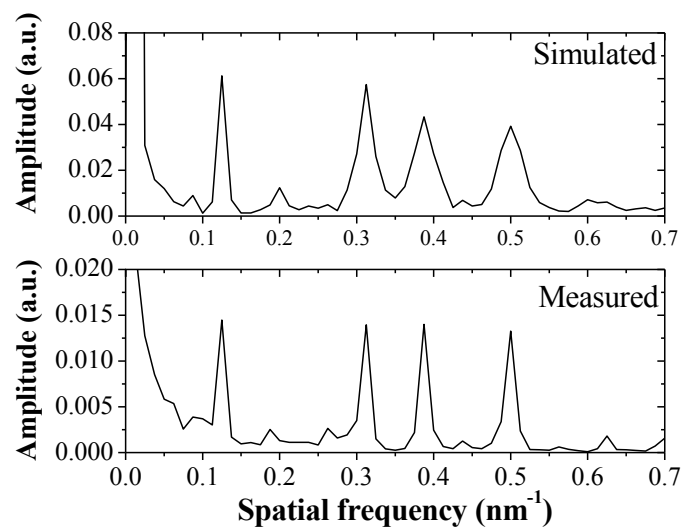

Fig. 4. Simulated and experimental results for the 2x8 HiBi FLM.

1 and $1.3 \mathrm{~m}$ were used. This HiBi fiber length is a crucial parameter to avoid the secondary contributions to be placed at the same spatial frequency than the main ones. Additionally, in order to compare the simulated results with the experimental measurements, the obtained transmission coefficient was superimposed to the spectrum profile of the optical interrogator used in the experiment.

As can be seen in Fig. 3, the spectral profile presents a complex wavelength behavior. Consequently, the monitoring of this interferometric sensors network by analyzing the wavelength maxima or minima would be very complicated.

However, the analysis of the signal by means of the Fourier transform is much easier. Figure 4 shows the simulated and measured FFT results for the interferometric multiplexing setup. The graphs show that the simulation fits with the experimental results since each interferometer contribution is located at the same frequency in both cases. It is worth noticing that amplitude values are for guidance only because attenuation introduced by the components was not taken into account for the simulation.

\section{B. Multi-section HiBi-FLM interferometer.}

The second multiplexing scheme is an additional HiBi-FLM structure which combines the contributions of the interpolarization phase shift by placing sections of HiBi fiber inside the fiber loop (Fig.5). In [14] this structure was theoretically analyzed as an optical filter providing the analytical results of the Jones matrix when two HiBi fiber sections are placed into the loop.

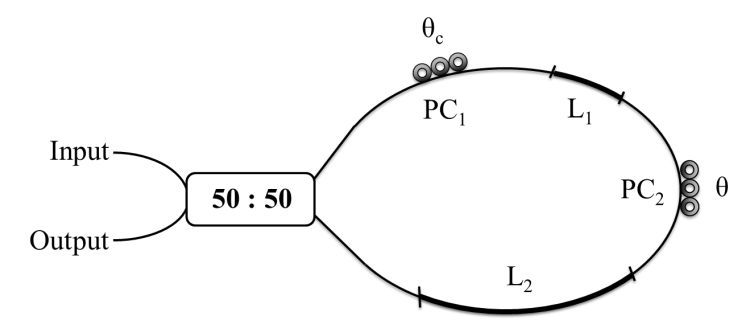

Fig. 5. Scheme of the multi-section HiBi-FLM interferometer.

The structure behavior is given as:

$$
\begin{aligned}
T= & {\left[\cos \left(\beta_{1}+\beta_{2}\right) \sin (\theta) \cos \left(\theta_{C}\right)+\right.} \\
& \left.+\cos \left(\beta_{1}-\beta_{2}\right) \cos (\theta) \sin \left(\theta_{C}\right)\right]^{2}
\end{aligned}
$$

Where:

$$
\beta_{n}=\left(2 \pi l_{n} \lambda_{0} / L_{B n} \lambda\right)
$$

and $L_{B n}$ is the beat length of the $n$-HiBi section at $\lambda_{0}$ wavelength, $\theta$ represents the angle of the polarization axis between the $\mathrm{HiBi}$ fibers (controlled by $\mathrm{PC}_{2}$ ) and $\theta_{c}$ defines the relative angle between the two ports of the coupler (controlled by $\mathrm{PC}_{1}$ ). Therefore, supposing a rotation angle between the $\mathrm{HiBi}$ fibers of $90^{\circ}$ and a correct alignment between the fibers with respect to fiber coupler, the achieved interference will be the combination of the two fibers contributions. In this manner the two-section HiBi loop interferometer encodes the sensor information in the phase of the FFT at the frequency that corresponds to the isolated interference created by each HiBi fiber section (each section has a different length).

To achieve the isolation of each interference contribution and a crosstalk-free operation, the angle of the polarization axis between the $\mathrm{HiBi}$ fibers must be $\theta=45^{\circ}$. This angle is adjusted using a polarization controller $\left(\mathrm{PC}_{2}\right)$ placed between the HiBi fiber sections. Additionally both polarization axes must be properly aligned to interfere coherently at the coupler using $\mathrm{PC}_{1}$ situated between the coupler and a $\mathrm{HiBi}$ fiber section. This polarization controller defines the relative angle $\theta_{c}$ between the two ports of the coupler. Figures 6 and 7 represent the simulated and experimental results obtained for different combinations of the angles between fibers. The first plot refers to the situation in which both $\mathrm{HiBi}$ fibers are misaligned $45^{\circ}$ between them and, although the ideal case would be $45^{\circ}, 57^{\circ}$ are set for matching the realistic situation obtained in the experimental results $\left(\theta=45 \theta_{C}=57\right)$. In this situation the interference contributions of the fiber sections $L_{1}$ and $L_{2}$ at the frequencies $f_{1}$ and $f_{2}$ are clearly seen. Additionally a contribution at the frequency $f_{2}-f_{1}$ can also be seen due to the misalignment. When the HiBi fibers are aligned between them and with the coupler $\left(\theta=\theta_{C}=0\right)$, there is no interference as depicted in Fig 6 (b) and 7 (b). Plots in (c) show the spatial frequency component referring to the situation at which the $\mathrm{HiBi}$ fibers are misaligned and aligned with the coupler $\left(\theta=90 \quad \theta_{C}=0\right)$. Finally, when the HiBi sections are aligned and both are misaligned with the coupler $\left(\theta=0 \quad \theta_{C}=90\right)$, the result is an interference equal to a fiber 


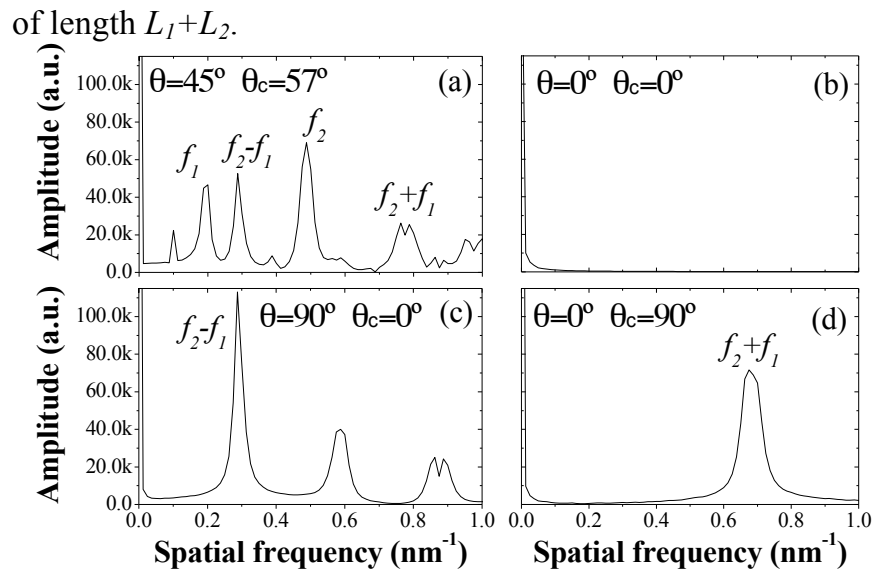

Fig. 6. Simulated results for the two-section HiBi loop interferometer.

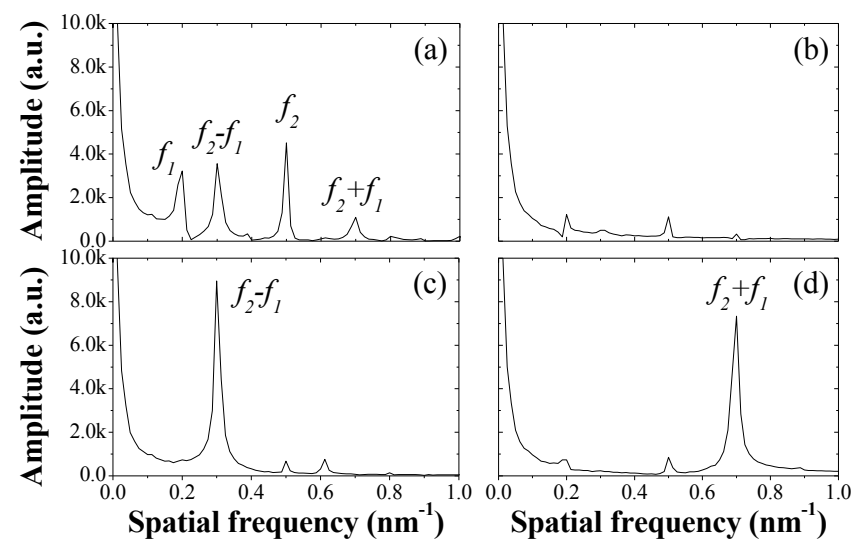

Fig. 7. Experimental results for the two-section HiBi loop interferometer.

After studying both multiplexing structures and their behavior, they were validated as sensor multiplexing schemes. Different parameters were taken into consideration: the sensor sensitivities, the crosstalk-free operation and the system instability which was used to calculate the system resolution. To perform these measurements, a commercial FBG interrogator (Smartec SM125) was used together with a custom software that was developed in order to analyze the FFT of the spectrum profile and to track the FFT phase of each sensor contribution.

\section{EXPERIMENTAL VERIFICATION}

In order to verify the proper operation of the proposed sensor-multiplexing schemes a series of experiments were carried out. Each section of HiBi fiber was considered as a sensor itself, sensitive to strain variations that were applied using a programmable translation stage with a resolution as good as $17 \mathrm{~nm}$. The $\mathrm{HiBi}$ fiber used is a polarization maintaining PCF (PM-1550-01 from NKT Photonics) with an attenuation of $1.0 \mathrm{~dB} / \mathrm{Km}$ and a $\sim 3.45 \mathrm{~mm}$ beat length. A photograph of its cross section can be seen in the inset of Figure 11. A commercial fiber-optic sensors interrogator (Smartec SM125) was used to monitor the optical signal reflected from the interferometers. This interrogator employs a tunable fiber laser with a scan frequency of $1 \mathrm{~Hz}$, a wavelength range from 1510 to $1590 \mathrm{~nm}$ and a dynamic range of $50 \mathrm{~dB}$. This device was initially designed for interrogating

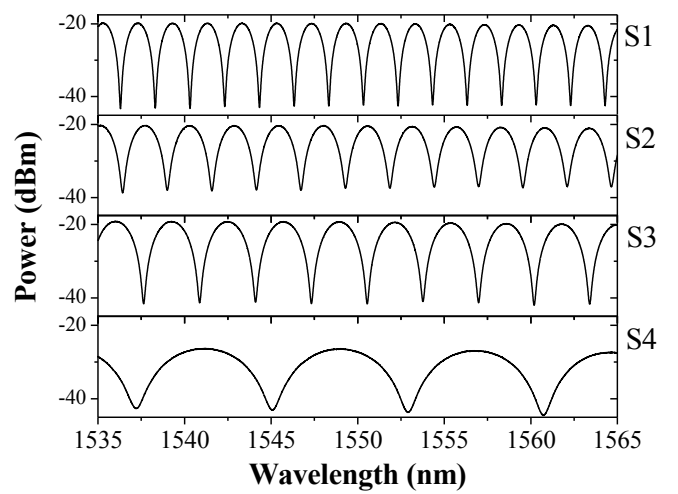

Fig. 8. Optical spectra of the four HiBi PCF sensors measured individually.

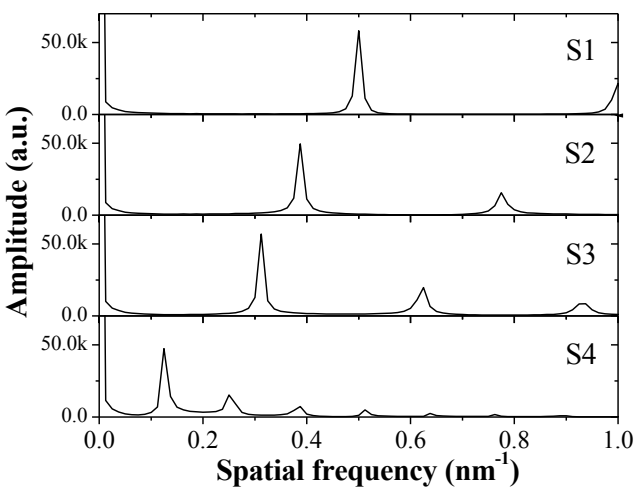

Fig. 9. FFT magnitude of the HiBi PCF sensors measured individually.

fiber Bragg grating (FBG) sensors, but by using the FFT its capabilities have been enhanced allowing interferometric multiplexing schemes to be interrogated as well. Both translation stages and the interrogator were controlled from a computer using custom software built upon Matlab. This application allowed to simultaneously control the translation stage and the interrogator parameters. Additionally, it displays the optical spectrum obtained by the interrogator and its FFT amplitude and phase every second. In order to measure the strain sensitivities of the sensors, the FFT phases measured at the frequencies corresponding to the peaks in the amplitude of the FFT had to be monitored.

Before performing the experiment, the sensing elements were analyzed in order to avoid frequency overlap between them. To avoid this effect, four different PCF fiber lengths of $1.3,1,0.8$ and 0.3 meters were used, corresponding to sensors 1 to 4 respectively. Every section has a different length since the characteristic spatial frequency of each interference contribution will depend on the fiber length. Consequently, as can be seen in Fig. 8 and 9 the amplitude peaks for the sensors 1 to 4 are placed at the spatial frequencies $0.5,0.3875,0.3125$ and $0.125 \mathrm{~nm}^{-1}$ respectively.

After measuring each sensor individually, as it has been previously pointed out, two different multiplexing schemes have been tested for sensing purposes.

\section{A. Parallel-multiplexed HiBi-FLMs using a $2 \times 8$ optical coupler}

In the first setup, using the configuration shown in Fig. 2, 
four FLMs were multiplexed using a 2x8 optical coupler.

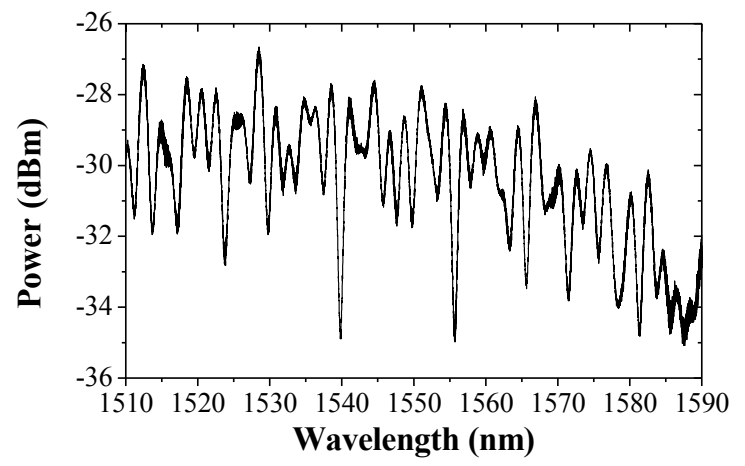

Fig. 10. Measured optical spectrum for the multiplexed 2x8 HiBi FLMs.

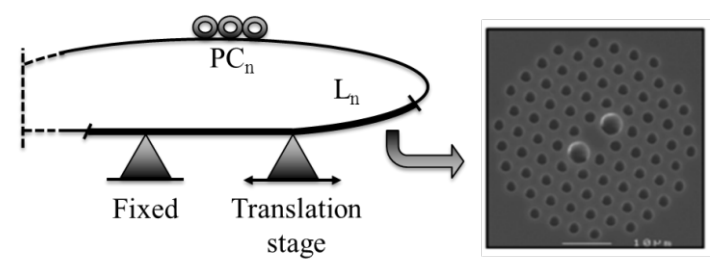

Fig. 11. Axial-strain test arrangement for a fiber loop mirror. HiBi PCF fiber used in the experiments.

These four different interference patterns mixed up in the coupler resulting in a received signal at the interrogator similar to the displayed in Fig. 10. As exposed before, by using the FFT analysis this signal can be decomposed unambiguously into four different amplitude peaks corresponding to the four different interference patterns.

In order to measure the strain sensitivities of the interferometers, a strain sweep was performed on each PCF section by means of the PC-controlled translation stage with a precision of $17 \mathrm{~nm}$. The experimental setup was adapted from the scheme shown in Fig. 2 by connecting the optical interrogator to the $2 \times 8$ coupler in which the four fiber loop mirrors were formed. A section of the HiBi fiber under test, considered as the axial-strain sensor, was fixed to the translation stage in order to perform the axial-strain sweep (Fig 11). Due to technical restrictions, the fixed length of the $\mathrm{HiBi}$ section was $16.6,21,15.8$ and $15.5 \mathrm{~cm}$ for sensors 1 to 4 respectively. As a result, the axial-strain applied to each sensor was different for a similar length increment, due to the different lengths of PCF fixed on the translation stage. Accordingly, a length increment of $4.233 \mu \mathrm{m}$ per step of the sweep was applied to each PCF section. This length increment supposed an axial-strain increment per step of 25.5, 20, 26.8 and $27 \mu \varepsilon$ for sensors 1 to 4 respectively. Figure 12 shows the variation of the FFT phase (at the frequencies corresponding to the peaks in the FFT amplitude) versus the strain applied to each sensor. As it was expected, the phase variation at the spatial frequencies $0.5,0.3875,0.3125$ and $0.125 \mathrm{~nm}^{-1}$ (sensors 1 to 4 ) exhibited a linear behavior. The corresponding sensitivities of the sensors 1 to 4 are $-0.155,-0.180,-0.154$ and $-0.145 \pi \mathrm{rad} / \mathrm{m} \varepsilon$ respectively showing a linear fitting error factor $\mathrm{R}^{2}$ of 0.9996 in the worst case.

In addition, a series of measurements were taken with the aim of studying the crosstalk between sensors. Figure 13 depicts the phase variation of each sensor when strain was

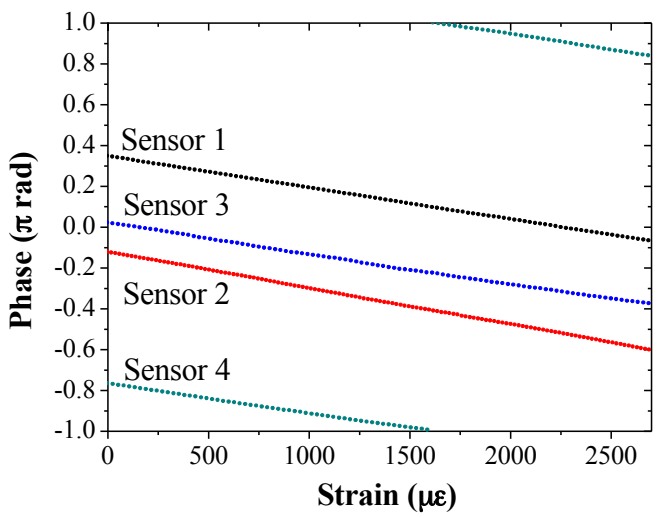

Fig. 12. Phase behavior of the sensors vs. applied strain.

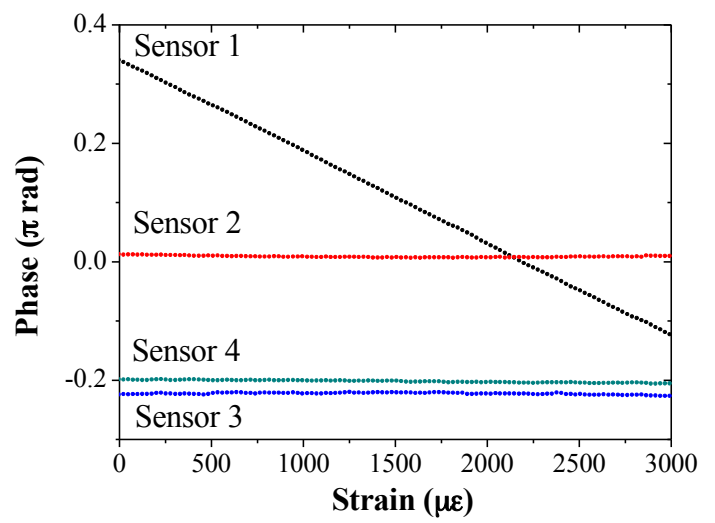

Fig. 13. Phase variation of the sensors when strain is applied to sensor 1.

applied just to the sensor 1 . It can be seen that sensors 2, 3 and 4 are not affected by the strain applied to sensor 1 . This test was repeated for every sensor obtaining the same results. Thus there was no appreciable crosstalk between sensors. This also implies that the phase noise given by spurious frequency contributions does not affect the sensor's performance.

\section{B. Multi-section HiBi-FLM interferometer}

The second proposed configuration was also verified for sensing purposes. In this case, the interferometer consisted of two $\mathrm{HiBi}$ sections and two polarization controllers (PCs) connected to a $2 \times 2$ coupler, as it is shown in Fig. 5. The HiBi fiber lengths used in this occasion for sensors 1 and 2 were 1.3 and $0.8 \mathrm{~m}$ respectively corresponding to the FFT spatial frequencies $f_{S I}=0.5 \mathrm{~nm}^{-1}$ and $f_{S 2}=0.3125 \mathrm{~nm}^{-1}$. As it has been explained in Section II, due to the interference properties of this scheme, extra frequencies are generated at frequencies $f_{S 1}+f_{S 2}=0.8125 \mathrm{~nm}^{-1}, f_{S 1}-f_{S 2}=0.1875 \mathrm{~nm}^{-1}$ (negative frequencies $-f_{S 1}-f_{S 2}$ and $-f_{S 1}+f_{S 2}$ are not taken into account). Depending on the PCs configuration, the influence of the extra frequencies $f_{S 1}+f_{S 2}$ and $f_{S 1}-f_{S 2}$ will change.

As an example, Fig. 15 shows the case in which the magnitude at $f_{S 1}-f_{S 2}$ is even higher than at $f_{S 1}$ or $f_{S 2}$. Additionally higher order spatial frequencies can be seen at other frequency combinations like $f_{S 2}-\left(f_{S 1}-f_{S 2}\right)=0.125 \mathrm{~nm}^{-1}$ or $f_{S 1}+\left(f_{S 1}-f_{S 2}\right)=0.6875 \mathrm{~nm}^{-1}$. As a consequence, when multiplexing a greater number of sensors these spatial frequencies should be avoided in order to get precise sensors 
reading.

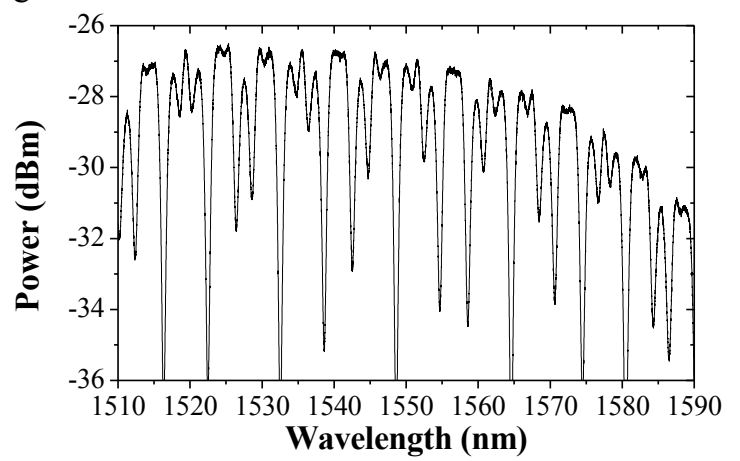

Fig. 14. Optical spectrum of the two-section HiBi FLM interferometer.
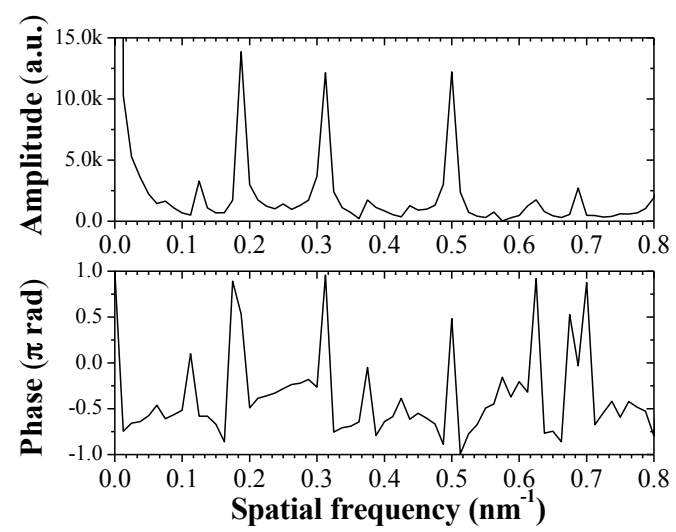

Fig. 15. FFT amplitude and phase of the two-section HiBi FLM interferometer.

As in the preceding case, the strain sensitivities of the sensors were measured by applying axial-strain to the two HiBi fiber sections. The applied strain varied from 0 to 3000 $\mu \varepsilon$ with a step of 25.5 and $26.8 \mu \varepsilon$ for sensors 1 and 2 . In this case Figure 16 and 17 show the FFT phase at the sensors (freq. 0.5 and $0.3125 \mathrm{~nm}^{-1}$ ) when strain was applied. It can be seen that the FFT phase of the strained sensor decreased linearly with strain with a sensitivity of -0.167 and $-0.155 \pi \mathrm{rad} / \mathrm{m} \varepsilon$ presenting fitting errors with a $\mathrm{R}^{2}=0.9994$ and 0.9998 respectively. In both cases the phase of the non-strained sensor remained steady evidencing that there is no crosstalk between sensors.

Additionally, spatial frequencies $f_{S 1}+f_{S 2}$ and $f_{S 1}-f_{S 2}$ have been depicted in order to confirm the theoretical analysis of the scheme. As expected, the phase variation at the spatial frequency $f_{S 1}+f_{S 2}=0.8125 \mathrm{~nm}^{-1}$ corresponds to the phase change of sensor 1 plus the phase variation of sensor 2 (7).

Similarly, the phase change at the spatial frequency $f_{S 1}-f_{S 2}=0.1875 \mathrm{~nm}^{-1}$ equals to the sensor 1 behavior minus the phase change of the sensor 2 (8).

$$
\begin{aligned}
& \Delta \phi\left(f_{S 1}+f_{S 2}\right)=\Delta \phi\left(f_{S 1}\right)+\Delta \phi\left(f_{S 2}\right) \\
& \Delta \phi\left(f_{S 1}-f_{S 2}\right)=\Delta \phi\left(f_{S 1}\right)-\Delta \phi\left(f_{S 2}\right)
\end{aligned}
$$

That is the reason why in Fig $16 \Delta \phi\left(f_{S 1}+f_{S 2}\right)=\Delta \phi\left(f_{S I}\right)$ since $\Delta \phi\left(f_{S 2}\right) \approx 0$. Equivalently in Fig. 17 can be seen that $\Delta \phi\left(f_{S_{1}}-f_{S_{2}}\right)$ behaves inversely to the phase of the sensor 2 since $\Delta \phi\left(f_{S I}\right) \approx 0$.

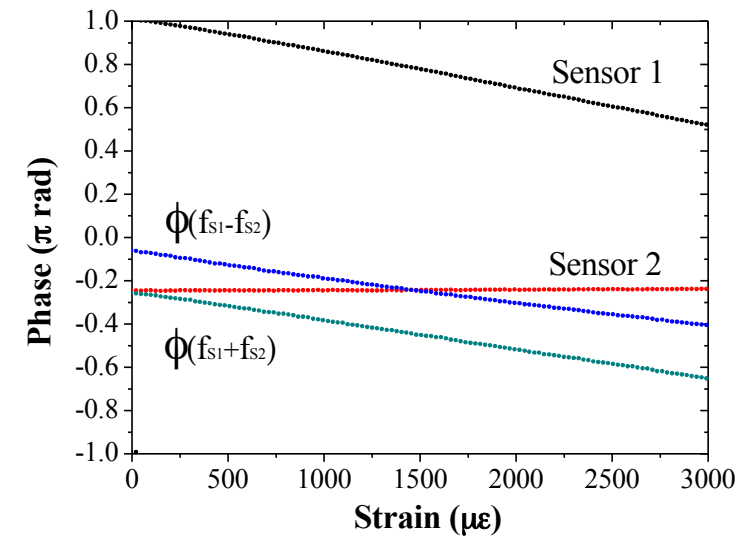

Fig. 16. Phase variation of the sensors when strain is applied to sensor 1.

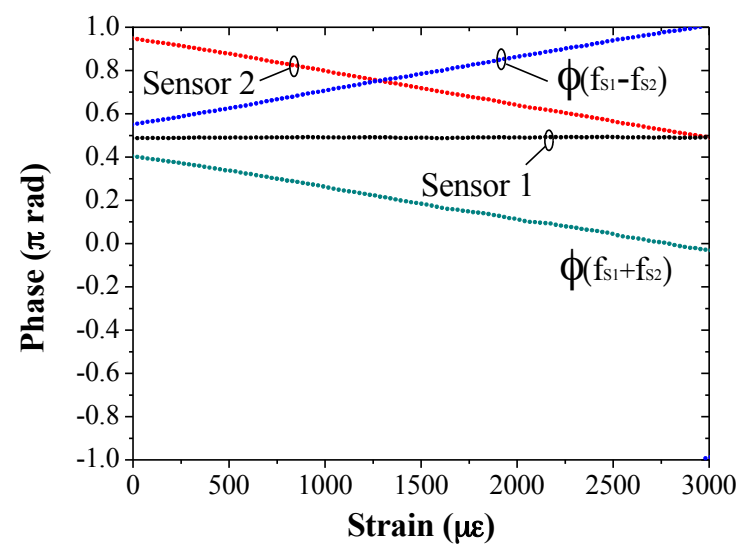

Fig. 17. Phase variation of the sensors when strain is applied to sensor 2 .

\section{General measuring concepts}

After validating the proposed configurations there are some general aspects that affect to both schemes. It is worth noticing that in Fig. 12, sensor 4 has a phase change from $-\pi$ to $\pi$ (must be taken into account the fact that a $\pi$ phase shift is equivalent to a $-\pi$ shift). This effect can be easily avoided by setting the initial value of the phase to value greater than $-0.45 \pi \mathrm{rad}$ using the polarization controller. In a worst-case scenario with a sensitivity of $-0.180 \pi \mathrm{rad} / \mathrm{m} \varepsilon$, the measurement range $2 \pi$ equals to more than $11000 \mu \varepsilon$. This value is over $5000 \mu \varepsilon$, the damage limit of this PCF fiber [15]. Therefore a $2 \pi$ phase range does not impose a limitation to the measurement system. Additionally, if needed, this phase change from $-\pi$ to $\pi$ can be easily handled by software.

In order to investigate the error on the measurements, a study of the phase instability against time was carried out. As shown in Fig. 18 the FFT phase of a sensor was monitored every second for 20 minutes. As a result, an instability factor of $1.25 \times 10^{-3} \pi \mathrm{rad}$ was obtained, equivalent to $6.9 \mu \varepsilon$ for a sensor sensitivity of $-0.180 \pi \mathrm{rad} / \mathrm{m} \varepsilon$. 


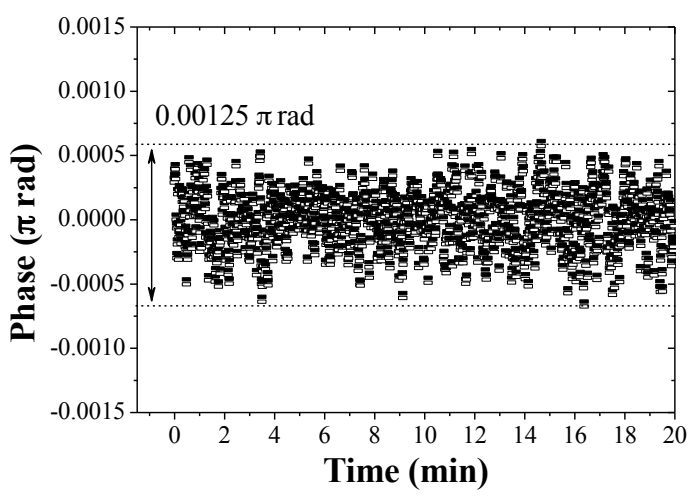

Fig. 18. FFT phase instability against time.

Authors would like to remark that the FFT analysis technique firstly proposed in [6] presents other advantages beyond its promising multiplexing capability. As the information is contained in the FFT phase, the amplitude of the interference is not as crucial as in other methods. Consequently authors estimate that this technique will allow a higher number of sensors to be multiplexed in a unique network without amplification. Under the realistic assumption of using a $2 \times 16$ coupler in a hybrid configuration of eight twosection FLM interferometers, 16 sensors can be multiplexed per interrogator channel. In the case of the Smartec SM125 interrogator used for the experiments, four channels are available, so we consider that $64 \mathrm{HiBi}$ sensors can be multiplexed without any difficulty by using the proposed schemes. The main restriction for multiplexing a greater number of sensors will reside in the frequency distribution of the sensors. A precise design of the spatial frequency location of the sensors should be done, requiring the use of longer lengths of PCF in order to work at higher frequencies. In the proposed study, the highest spatial frequency was at freq. 0.5 $\mathrm{nm}^{-1}$, corresponding to a length of $1.3 \mathrm{~m}$. However, the FFT spatial frequency limit is $100 \mathrm{~nm}^{-1}$, which is imposed by the 16000 points given by the interrogator.

\section{CONCLUSION}

In conclusion, a theoretical and experimental study of two interferometric sensor multiplexing schemes has been carried out by means of the FFT analysis. Both structures are based on fiber loop mirrors that included sections of HiBi fiber that operated as sensors. The first scheme multiplexed four interferometric sensors in a $2 \times 8$ optical coupler and the second included two sections of $\mathrm{HiBi}$ fiber in a single FLM interferometer.

A theoretical analysis has been performed where simulations agreed with the experimental results. Due to the use of the fast Fourier transform, these multiplexing schemes can be properly interrogated by monitoring the FFT phase change at the characteristic spatial-frequency of each sensor. Additionally, both multiplexing structures were validated performing a strain sweep for every sensor. For this purpose a commercial interrogator and a custom Matlab program were used to compute the FFT and to monitor the FFT phase change in real-time $(1 \mathrm{~Hz})$. Sensitivities between -0.180 and $-0.145 \pi$ $\mathrm{rad} / \mathrm{m} \varepsilon$ were attained with a fitting factor $\mathrm{R}^{2}=0.9994$ in the worst case. An instability study was also performed showing a maximum measurement error of $6.9 \mu \varepsilon$. Finally, the freecrosstalk operation between sensors was proven.

The use of the FFT analysis addresses one of the main drawbacks of PCF sensors, allowing its multiplexing and realtime monitoring using a commercial optical FBG sensors interrogator, combined with a simple and precise measurement technique. Moreover, due to the use of the FFT phase as the sensing parameter, power constraints are not as limiting as in other techniques, allowing more sensors to be multiplexed. On the other hand, it must be remarked that multiplexing a large number of interferometric sensors using this topologies, although possible, would require a detailed study of the spatial frequency allocation of the sensors.

\section{REFERENCES}

[1] X. Fang, H. Ji, C. T. Allen, K. Demarest and L. Pelz. (1997, April). A Compound High-Order Polarization-Independent Birrefringence Filter Using Sagnac Interferometers. IEEE Photonics Technology Letters, 9(4), $458-460$

[2] A. M. R. Pinto, M. Bravo, M. Fernadez-Vallejo, M. Lopez-Amo, J. Kobelke and K. Schuster. (2011). Suspended-core fiber Sagnac combined dual-random mirror Raman fiber laser. Optics Express, 19(12), 11906-11915.

[3] M. Bravo, A. M. R. Pinto, M. Lopez-Amo S. Rota-Rodrigo, I. Ibañez, Lopez-Amo, J. Kobelke and K. Schuster. (2012, January). High precision micro-displacement fiber sensor through a suspended-core Sagnac interferometer. Optics Letters, 37(2), 202-204.

[4] A.M.R. Pinto and Lopez-Amo, M. (2012). Photonic crystal fibers for sensing applications. Journal of Sensors, art. no. 598178

[5] O. Frazão, J. M. Baptista and J. L. Santos. (2007, November). Recent Advances in High-Birefringence Fiber Loop Mirrors Sensors. Sensors, 7, 2970-2983.

[6] D. Barrera, J. Villatoro, V. P. Finazzi, G. A. Cardenas-Sevilla, V. P. Minkovich, S. Sales, V. Pruneri, (2010, December). Low-Loss Photonic Crystal Fiber Interferometers for Sensor Networks. Journal of Lightwave Technology, 28(24), 3542-3547.

[7] M. Bravo, M. Fernández-Vallejo, M. Echapare, M. López-Amo, J. Kobelke and K. Schuster, (2013). Multiplexing of six microdisplacement suspended-core Sagnac interferometer sensors with a Raman-Erbium fiber laser. Optics Express, 21 (3), pp. 2971-2977.

[8] G. A. Cárdenas-Sevilla, V. Finazzi, J. Villatoro, and V. Pruner, (2011). Photonic crystal fiber sensor array based on modes overlapping. Opt. Express 19(8), 7596-7602.

[9] H. Y. Fu, A. C. L. Wong, P. A. Childs, H. Y. Tam, Y. B. Liao, C. Lu, and P. K. A. Wai, (2009). Multiplexing of polarization-maintaining photonic crystal fiber based Sagnac interferometric sensors. Opt. Express 17(21), 18501-18512.

[10] B. Culshaw (2005, November). The optical fiber Sagnac interferometer: an overview of its principles and applications. Measurement Science and Technology, 17, R1-R16.

[11] G. Sagnac (1913) L'éther lumineux d'emontré par l'effet du vent relatif d'éther dans un interféromètre en rotation uniforme. C. R. Acad. Sci. 95, 708-710.

[12] D. B. Mortimore. (1988, July). Fiber Loop Reflectors. Journal of Lightwave Technology, 6 (7) 1217-1224

[13] L. Liu, Q. Zhao, G. Zhou, H. Zhang, S. Chen, L. Zhao, Y. Yao, P. Guo and X. Dong, (2004, March). Study on an optical filter constituted by concatenated $\mathrm{Hi}-\mathrm{Bi}$ fiber loop mirrors. Microwave and Optical Technology Letters, 43 (1), 23-26.

[14] Y. Liu, B. Liu, X. Feng, W. Zhang, G. Zhou, S. Yuan, G. Kai and X. Dong, (2005, April). High-birefringence fiber loop mirrors and their applications as sensors. Applied Opticst. 44 (12) 2382-2390.

[15] S. Rota-Rodrigo, A.M. Pinto, M. Bravo and M. Lopez-Amo, (2013). An in-reflection strain sensing head based on a Hi-Bi photonic crystal fiber. Sensors (Basel, Switzerland), 13 (7), pp. 8095-8102. 